Journal of Case Reports 2018;8(4):229-231

\title{
Intra-operative Pulmonary Embolism during Laparoscopic Colectomy for Crohn Colitis
}

\author{
Michael Koussa, Petachia Reissman \\ Department of Surgery, Shaare-Zedek Medical Center, The Hebrew University School of Medicine, Jerusalem, Israel.
}

\section{Corresponding Author:}

Michael Koussa

Email: michael.j.koussa@gmail.com

This is an Open Access article distributed under the terms of the Creative Commons Attribution License (creativecommons.org/ licenses/by/3.0).

Received Accepted

Published

February 17, 2018

September 21, 2018

October 5, 2018

\begin{abstract}
Background: Intra-operative pulmonary embolism $(\mathrm{PE})$ is a rare but fatal complication leading to sudden intra-operative cardiovascular collapse with most cases being diagnosed by post mortem. Accurate diagnosis is often difficult as other more common causes of cardiovascular collapse are considered. Real time Transesophageal Echocardiography (TEE) in addition to commonly used intra-operative anesthesia monitoring is a key modality in aiding immediate diagnosis allowing for the appropriate critical care. Case Report: We report an unusual case of intra-operative PE during elective laparoscopic colectomy in a patient suffering from Crohn's colitis, diagnosed by intra-operative TEE. Post-operative CT pulmonary angiogram confirmed the diagnosis. Intensive supportive treatment led to regaining hemodynamic stability and was followed by complete recovery. Conclusion: This case report is unique in several aspects which will be discussed, but most importantly should raise awareness amongst all care givers of inflammatory bowel disease patients who undergo major surgery after prolonged pre-operative hospitalization with increased risk of thrombo-embolic complications.
\end{abstract}

Keywords: Angiography, Critical Care, Inflammatory Bowel Disease, Laparoscopy, Pulmonary Embolism, Transesophageal Echocardiography.

\section{Introduction}

Peri-operative thrombo-embolism may be related to many factors including: hyper-coagulability, malignancy, prolonged immobilization, heavy smoking, pregnancy, hormonal and other medications [1,2]. The usual presentation is of post-operative symptomatic deep venous thrombosis (DVT) or pulmonary embolism (PE). Intra-operative PE (IOPE) is unusual, and its risk factors include mainly orthopedic surgery and trauma patients due to limb manipulation following a period of immobilization [1-5]. Massive IOPE with sudden cardiovascular collapse is extremely rare; its diagnosis may be difficult and in most cases will lead to death. Alongside other anesthesia monitoring if used intra-operatively, transesophageal echocardiography (TEE), may visualize the thrombus as well as acute right ventricular dilatation and failure [1,2,6,7]. We report an unusual case of IOPE detected by TEE, during laparoscopic extended right colectomy for transverse colonic stricture due to long standing Crohn's colitis. Immediate accurate diagnosis and intensive care lead to complete recovery. Possible causes and potential preventive measures will be discussed.

\section{Case Report}

A 61 year old male patient with Crohn's colitis diagnosed 20 years ago, whom was not under regular follow up nor received regular treatment, was admitted through the emergency department with chronic large bowel obstruction due to a tight stricture in the mid transverse colon, and a general long lasting deterioration with weight loss and poor nutrition. CT scan showed the stricture 
with moderate dilatation of the right colon and small bowel. Mild colitis was seen in the left and sigmoid colon. Colonoscopy revealed the same findings and even a pediatric scope was unable to pass the stricture. Due to his poor condition, preoperative treatment was started with total parenteral nutrition after failed trial of elemental nutrition and prophylactic dose of low molecular weight heparin was started on admission and administered daily. After three weeks of admission and improved condition, the patient was taken to surgery with planned laparoscopic extended right colectomy and ileostomy due to persistent left colitis. At surgery, the patient was initially completely stable, but after approximately one hour, following the division of the left transverse colon without any surgical difficulties or manipulations, a sudden and severe drop in his blood pressure to systolic $40 \mathrm{mmHg}$ occurred, bradycardia of 48 beats per minute followed by tachycardia of 130 beats per minute, abrupt reduction in end tidal $\mathrm{CO}_{2}$ to $17 \mathrm{mmHg}$ and saturation to $86 \%$ and evidence of right ventricular strain was associated on the three lead electrocardiogram. Immediate TEE (the instrument is always standby in our OR in all major cases) showed right ventricle dilatation with a clot in the lumen at its outlet. Within three to four minutes the clot disappeared and the right ventricle returned to normal size and function. Parallel to the TEE, immediate noradrenaline treatment and full anticoagulation was started along with $100 \%$ of oxygen and fluid administration. Within 1015 minutes. the patient regained hemodynamic stability, the operation was quickly concluded with creation of an end ileostomy and mucous fistula of the left transverse colon as planned.

The patient was transferred to the intensive care unit for further treatment and observation. On the next day, after remaining stable with gradual discontinuation of noradrenaline support, a CT pulmonary angiogram was performed; multiple small peripheral clots in sub segmental arteries of the right and left lower lung lobes were observed.
Doppler ultrasound of lower extremities did not show residual clot. On post-operative day three the patient was transferred to the ward, followed by an uneventful recovery and discharged home on the $10^{\text {th }}$ post-operative day.

\section{Discussion}

Intra-operative pulmonary embolism is rarely diagnosed and in most reported cases the diagnosis was made by post mortem (PM) after fatal outcome. Its diagnosis may be difficult as other more common causes of sudden cardiovascular collapse are considered and the lack of real time intra-operative TEE which is the best intra-operative diagnostic modality. Therefore, this complication is probably under reported. We here present an unusual case of IOPE with a favorable outcome. Our case is unique in several aspects like: immediate intra-operative diagnosis, patient's survival, and Crohn's disease as a risk factor especially when surgery follows a prolonged hospitalization. We believe that the patho-physiology of IOPE in this case was related to the prolonged hospital stay prior to the surgical procedure, with somewhat limited mobilization, along with IBD which is a known hyper-coagulable state, led to the creation of DVT dislodged during surgery Interestingly, the patient did receive prophylactic low molecular weight heparin during his pre-operative stay and did not have any clinical signs or symptoms of DVT prior to surgery. IBD patients, who are in poor nutritional condition, often require prolonged hospitalization for nutritional support before surgery can be safely performed, and therefore are at specially increased risk.

We believe that although prophylactic anticoagulation was given, a pre-operative Doppler ultrasound of lower extremities should have been performed to rule out DVT. If DVT would have been diagnosed, pre-operative insertion of IVC filter could have prevented such a potentially fatal complication. Immediate diagnosis by TEE in the OR, allowed for immediate and appropriate response by the anesthesia team leading to the patient's favorable outcome. 


\section{Conclusion}

IOPE is a rare but fatal complication. TEE is the main diagnostic modality and should be part of the anesthetist's armamentarium in any major surgery of a high risk patient. IBD patients who undergo surgery after prolonged hospital stay should be considered high risk for IOPE and therefore should be studied for the presence of deep vein thrombosis regardless of clinical signs or symptoms, and if present, an inferior vena cava filter should be considered.

Contributors: MK: designed and drafted the manuscript; PR: revising it critically for important intellectual content; MK will act as a guarantor. All authors approved the final version of this manuscript.

Funding: None; Competing interests: None stated.

\section{References}

1. Desciak MC, Martin DE. Perioperative pulmonary embolism: diagnosis and anesthetic management. J Clin Anesthesia. 2011;23:153-165.
2. Sakon M, Kakkar AK, Ikeda M. Sekimoto M, Nakamori $\mathrm{S}$, Yano M, et al. Current status of pulmonary embolism in general surgery in Japan. Surg Today. 2004;34:805810 .

3. Tortosa JA, Hernandez-Palazon J. Fatal massive intraoperative pulmonary embolism while placing a patient in the surgical position (letter). Eur J Anesthesiology. 1999;16:350.

4. Aitchison S, Hoopes CW, Roth JS. Venovenous extracorporeal membrane oxygenation for the treatment of acute pulmonary embolism after ventral hernia repair. Am Surgeon. 2013;79:444-446.

5. Jain A, Pradhan S, Jain S, Chakraborty A, Ranjan P. Fatal intra-operative pulmonary embolism in a child - a case report. Ped Anesthesia. 2009;19:286-287.

6. Ju H, Wong SSF, Bertram SG. Intra-operative pulmonary thrombus diagnosed by transesophageal echocardiography. Echocardiography. 2013;30:12321233.

7. Rosenberger P, Shernan SK, Body SC, Eltzschig HK. Utility of intraoperative transesophageal echocardiography for diagnosis of pulmonary embolism. Anesth Analg. 2004;99:12-16. 